\title{
MAXIMUM ANTICHAINS: A SUFFICIENT CONDITION
}

\author{
MICHAEL J. KLASS ${ }^{1}$
}

ABSTRACT. Given the finite partially ordered set $(Q, \leq)$, one might wish to know whether a maximal (nonextendible) antichain is a maximum antichain. Our result generalizes a theorem of Baker, which in turn constitutes a generalization of Sperner's lemma.

Introduction. Given a finite partially ordered set $(Q, \leq)$ and a maximal antichain $A$, one might seek conditions which force $A$ to be a maximum antichain of $Q$. In this note we find some such conditions.

Sperner's lemma [3] states that a maximum antichain of the subset lattice of an $n$-element set consists of all the points at the $[n / 2]$ level. Our result implies both Sperner's lemma and a generalization thereof given by Baker [1]. Baker's result states that in a finite partially ordered set with a rank function, if the number of covering and covered elements is constant for elements of any given rank, then the elements at the most populous level constitute a maximum antichain.

Notation and Terminology. $1 .(Q, \leq)$ denotes a finite partially ordered set.

2. Let $a_{1}, a_{2} \in Q$. If $a_{1}$ and $a_{2}$ are comparable, we write $a_{1} R a_{2}$ (for " $a_{1}$ is related to $a_{2}$ "); otherwise we write $a_{1} k_{2} a_{2}$.

3. A nonempty subset $S$ of $Q$ is a chain (antichain) iff, for any two distinct elements $a_{1}, a_{2}$ of $S, a_{1} R a_{2}\left(a_{1} R a_{2}\right)$.

4. $S$ is a maximal chain (antichain) iff, for any $a \in Q-S, S \cup\{a\}$ is not a chain (antichain).

5. $S$ is a maximum chain (antichain) iff, for any other chain (antichain) $T,|S| \geq|T|$.

6. For any nonempty subset $S$ of $Q$, let $M(S)$ denote the collection of maximal chains of $Q$ which intersect $S$.

Received by the editors January 18, 1973 and, in revised form, June 14, 1973. AMS (MOS) subject classifications (1970). Primary 06A10; Secondary 06A20.

Key words and phrases. Maximum antichains, Sperner's lemma, partially ordered sets.

1 Supported by the Fannie and John Hertz Foundation. 
Observe that if $S=\left\{q_{1}, \cdots, q_{n}\right\}$ is an antichain, then $M(S)$ is the disjoint union of $M\left(q_{j}\right)$ for $j=1,2, \cdots, n$.

Theorem. Let $\left\{q_{1}, \cdots, q_{n}\right\}$ be a maximal antichain of $Q$. Assume that

(i) for each $q \in Q$ and $j \leq n \ni q R q_{j},\left|M\left(q_{j}\right)\right| \leq|M(q)|$, and

(ii) $|M(Q)| \leq\left|\bigcup_{i=1}^{n} M\left(q_{i}\right)\right|+1$.

Then $\left\{q_{1}, \cdots, q_{n}\right\}$ is a maximum antichain.

Proof. Let $\left\{p_{1}, \cdots, p_{m}\right\}$ be an antichain of $Q$. We show that $n \geq m$. For $i \leq m$, let $Q_{i}=\left\{q_{j}: q_{j} R p_{i}\right\} .\left\{q_{1}, \cdots, q_{n}\right\}$ is a maximal antichain so $Q_{i} \neq \varnothing$ for $i \leq m$. Assume $\left\{Q_{1}, \cdots, Q_{m}\right\}$ does not have a system of distinct representatives (SDR). There is a smallest subcollection, say $\left\{Q_{1}, \cdots, Q_{k+1}\right\}$, which does not have an SDR. (Here $1 \leq k<m$.) It follows easily from the theory of SDR's [2] that $\left|\bigcup_{i=1}^{k} Q_{i}\right|=k$ and that $Q_{k+1} \subseteq \bigcup_{i=1}^{k} Q_{i}$. To see this observe:

(a) $\left|\bigcup_{i \in F_{0}} Q_{i}\right|<\left|F_{0}\right|$ for some $F_{0} \subseteq\{1,2, \cdots, k+1\}$ since $\left\{Q_{1}, \cdots\right.$, $\left.Q_{k+1}\right\}$ does not have an SDR.

(b) $\left\{Q_{i}: i \in F\right\}$ has an $\operatorname{SDR}$ if $|F| \leq k$ and $F \subseteq\{1,2 .::, m\}$.

(c) From (b) it follows that $\left|\bigcup_{i \in F} Q_{i}\right| \geq|F|$ if $|F| \leq k$.

(d) Consequently, $F_{0}=\{1,2, \cdot: \cdot, k+1\}$ and $k \leq\left|\bigcup_{i=1}^{k} Q_{i}\right| \leq\left|\bigcup_{i=1}^{k+1} Q_{i}\right|$ $<k+1$.

Relabelling if necessary, we may suppose $q_{1}, \cdots, q_{k}$ is an SDR for $\left\{Q_{1}, \cdots, Q_{k}\right\}$, where $q_{i} \in Q_{i}$ for $i \leq k$. Thus, for $i \leq k, p_{i} R q_{i}$ and so $\left|M\left(q_{i}\right)\right| \leq\left|M\left(p_{i}\right)\right|$.

All but at most one maximal chain which intersects $\left\{p_{1}, \cdots, p_{k+1}\right\}$ intersects $q_{j}$ for some $j \leq n$. Whenever there is such an intersection, $p_{l} R q_{j}$ for some $l \leq k+1$ which then implies that $q_{j} \in \bigcup_{i=1}^{k+1} Q_{i}$ and therefore that $j \leq k$. Consequently,

$$
\begin{aligned}
&\left|M\left(\left\{p_{1}, \cdots, p_{k+1}\right\}\right)\right|-1 \leq\left|M\left(\left\{q_{1}, \cdots, q_{k}\right\}\right)\right| . \\
&\left|M\left(\left\{p_{1}, \cdots, p_{k+1}\right\}\right)\right|=\left|\bigcup_{i=1}^{k+1} M\left(p_{i}\right)\right|=\sum_{i=1}^{k+1}\left|M\left(p_{i}\right)\right| \geq \sum_{i=1}^{k}\left|M\left(q_{i}\right)\right|+\left|M\left(p_{k+1}\right)\right| \\
&=\left|M\left(\left\{q_{1}, \cdots, q_{k}\right\}\right)\right|+\left|M\left(p_{k+1}\right)\right| \\
& \geq\left|M\left(\left\{p_{1}, \cdots, p_{k+1}\right\}\right)\right|-1+\left|M\left(p_{k+1}\right)\right| \Rightarrow\left|M\left(p_{k+1}\right)\right| \leq 1 .
\end{aligned}
$$

There exists an $i \leq k \ni q_{i} R p_{k+1}$. Then $1 \leq\left|M\left(q_{i}\right)\right| \leq\left|M\left(p_{k+1}\right)\right| \leq 1$. We also have $q_{i} R p_{i}$. Since there is only one maximal chain through $q_{j}, p_{i} R p_{k+1}$ $\Rightarrow \Leftarrow$ contradiction. Hence $\left\{Q_{1}, \cdots, Q_{m}\right\}$ has an $\operatorname{SDR}$ and so $n \geq m$. 
An example constructed with the aid of Paul Klembeck shows that hypothesis (ii) cannot be deleted. A diagram of the example is as follows:

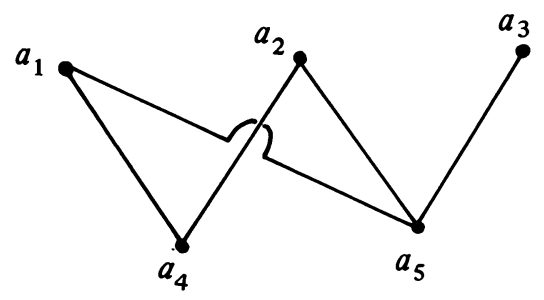

$$
\begin{aligned}
& a_{1} \geqslant a_{4}, a_{1} \geqslant a_{5}, \\
& a_{2} \geqslant a_{4}, a_{2} \geqslant a_{5}, \\
& a_{3} \geqslant a_{5} .
\end{aligned}
$$

The points $a_{3}$ and $a_{4}$ constitute a maximal antichain satisfying (i). However, $\left\{a_{1}, a_{2}, a_{3}\right\}$ is the maximum antichain.

We indicate how this theorem implies the result of Baker. In Baker's theorem, each point $q \in Q$ at level $l$ lies below $b_{l}$ points at level $l+1$, while in turn covering $a_{l}$ points at level $l-1$. A direct argument counting the number of maximal chains passing through a given point at, say, level $l$ shows that any two points at level $l$ have the same number of maximal chains passing through them. Also, note that every maximal chain of $Q$ passes through some point of level $l$. Therefore, the number of maximal chains passing through a given point at level $l$ is $M / p_{l}$, where $M$ is the total number of maximal chains of $Q$ and $p_{l}$ is the number of points of $Q$ at level $l$. If we therefore let $\left\{q_{1}, \cdots, q_{n}\right\}$ be the collection of points of $Q$ at the most populous level, this set will be a maximal antichain of $Q$ satisfying conditions (i) and (ii).

\section{REFERENCES}

1. K. A. Baker, A generalization of Sperner's lemma, J. Combinatorial Theory, 6(1969), 224-225. MR 38 \# 4368.

2. M. Hall, Jr., Combinatorial theory, Blaisdell, Waltham, Mass., 1967, p. 45. MR 37 \#8.

3. E. Sperner, Ein Satz über Untermengen einer endlichen Menge, Math. Z. 27(1928), 544-548.

DEPARTMENT OF MATHEMATICS, UNIVERSITY OF CALIFORNIA, LOS ANGELES, CALIFORNIA 90024 .

Current address: Department of Mathematics, California Institute of Technology, Pasadena, California 91109 